\title{
On Optimal AUV Track-Spacing for Underwater Mine Detection
}

\author{
David P. Williams
}

\begin{abstract}
This work addresses the task of designing the optimal survey route that an autonomous underwater vehicle (AUV) should take in mine countermeasures (MCM) operations. It is assumed that the AUV is equipped with a side-looking sonar that is capable of generating high-resolution imagery of the underwater environment. The objective of the path-planning task is framed in terms of maximizing the success of detecting underwater mines in such imagery. Several commonly made - but inaccurate - assumptions about the problem are raised and refuted; it is demonstrated that mine detection performance depends on both range and seabed type. The issue of how to update detection probabilities when multiple views are obtained is also addressed. These various considerations are exploited in conjunction with synthetic aperture sonar (SAS) data to predict detection performance and efficiently design AUV routes that outperform standard ladder surveys. The proposed algorithm can be used to assess and quantify detection performance achieved in past, as well as future, missions. Because the entire route of the AUV can still be designed before deployment, no additional onboard processing or adaptive capabilities are required of the AUV. Therefore, the proposed approach can be immediately applied to systems conducting MCM operations at sea. The method is demonstrated on real SAS imagery collected by an AUV in the Baltic Sea.
\end{abstract}

\section{INTRODUCTION}

This work addresses the task of designing the optimal survey route that an autonomous underwater vehicle (AUV) should take in mine countermeasures (MCM) operations. It is assumed that the AUV is equipped with a side-looking sonar that is capable of generating high-resolution imagery of the underwater environment. The objective of the pathplanning task is framed in terms of maximizing the success of detecting underwater mines in such imagery.

Most research about path planning for AUVs deals with attempting to find a route that will allow an AUV to transit safely from one location to another, or through a series of waypoints (e.g., [1]-[4]). With the exception of the water currents that exist in the underwater milieu, this objective is largely the same as for path planning with ground-based robots. That is, the issue of AUV path-planning can essentially be treated as an obstacle avoidance problem (albeit in three-dimensional space) with additional robot control constraints.

Path planning when specifically concerned with MCM operations, however, is markedly different because the route that the AUV takes will dictate the data that is collected and subsequently analyzed. To paraphrase a cliché about life, the emphasis and value in MCM operations with an AUV lie not on the destination, but rather on the journey.

D. Williams is with the NATO Undersea Research Centre, $19126 \mathrm{La}$ Spezia (SP), Italy. williamsenurc.nato.int
Moreover, in MCM applications, the area of interest that must be surveyed is typically a large rectangular area that is also generally free of obstacles. In this regard, the MCM problem shares more similarities to space-based applications - such as spatial coverage using orbiting satellites [5] or rovers [6] - than to the general problem of path planning in underwater environments.

The path-planning approach currently used in practice for underwater mine detection operations is to design a simple ladder survey with equidistant tracks over the mission area. This ladder survey is also referred to as a line sweep, a lawnmower pattern, seed sowing, and a boustrophedon ${ }^{1}$ path.

The adherence to traversing parallel tracks in MCM operations is partly because the collected raw data is subsequently processed into imagery (e.g., SAS imagery), for which such data is preferable. Thus, the crux of the path-planning task becomes how to design the spacing of (parallel) tracks.

\section{A. Previous Work}

The popularity and perpetuation of the simplistic laddersurvey of equidistant tracks can be attributed, in part, to several inaccurate assumptions made about underwater mine detection. The most egregious assumptions made in previous work about path planning for mine detection is the manner in which the effects of range, seabed type, and multiple views (on detection probabilities) are handled.

In [8], [9], it is assumed (incorrectly) that mine detection is independent of both range and seabed type. In [10], it is also assumed that mine detection is independent of range, but an ad hoc dependence on seabed type is offered. Specifically, in [10], the probability of detection is assumed to be a ternary quantity in which the values $0,0.5$, and 1 are assigned to regions that (i) have not been interrogated, (ii) consist of sand ripples or cluttered seabed, and (iii) consist of (flat) sandy seabed, respectively.

In sonar imagery, mines (as well as other objects raised above the seafloor) exhibit a characteristic highlight-shadow pattern that is commonly used for detection purposes. These characteristics are affected by the range of the object from the sensor, which in turn implies that detection performance is also dependent on range. At longer ranges, less energy is returned to the receiver, making the highlight portion of the mine weaker (and thus more difficult to detect). Similarly, the geometry of the problem means that a mine located at

\footnotetext{
${ }^{1}$ Strictly speaking, the term "boustrophedon" defines only writing that alternates in direction on consecutive lines; nevertheless, the term has been coopted (initially in [7]) to describe the standard survey patterns of AUVs as well.
} 


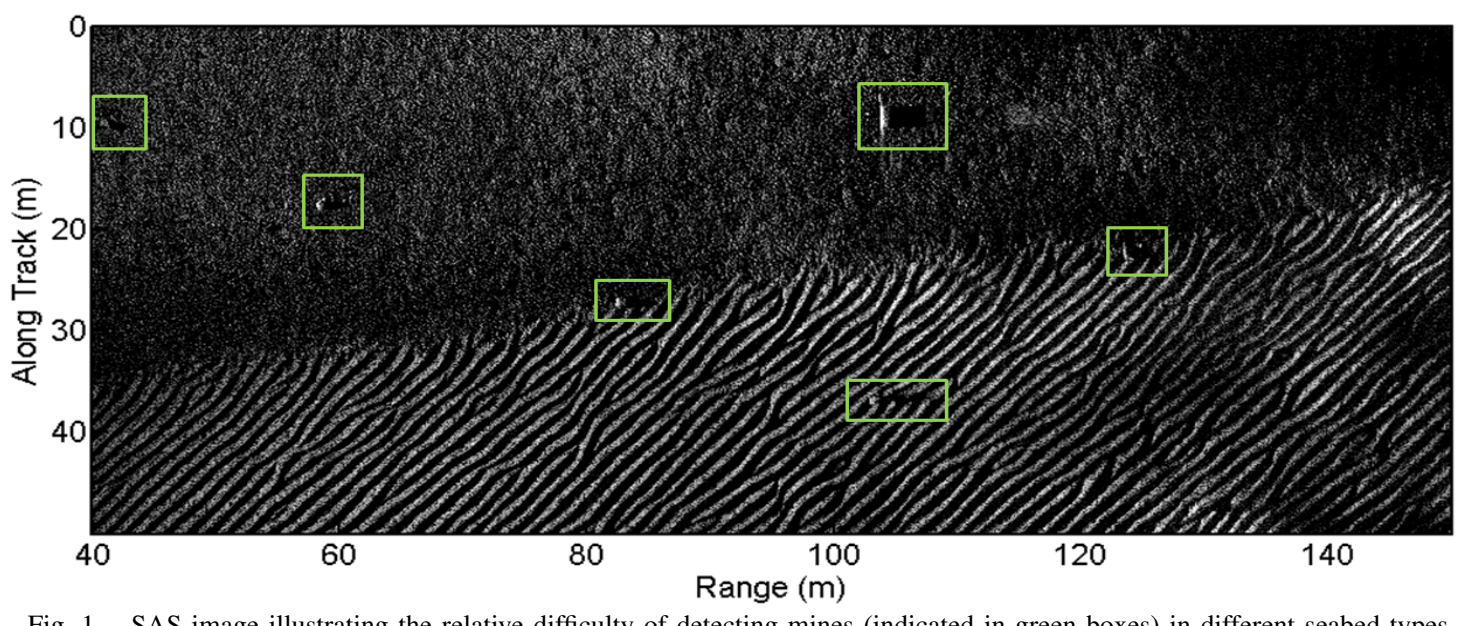

Fig. 1. SAS image illustrating the relative difficulty of detecting mines (indicated in green boxes) in different seabed types.

short range will have a short shadow because of the steep grazing angle.

Mine detection performance is also strongly dependent on seabed type. For example, in seabed characterized by sand ripples, a mine can blend in with the highlight of ripples or be hidden in the shadows cast by ripples. In contrast, because flat seabed does not introduce such complications, detection performance should reflect this fact. Example SAS imagery illustrating the effects of seabed type and range on detection are shown in Fig. 1.

In [8], the detector is assumed to be perfect, resulting in a binary performance in which the probability of detection ${ }^{2}$ is 1 for areas covered (i.e., by the sonar swath), and 0 for areas not covered. This assumption of a perfect detector is unjustified, and precludes addressing the problem of how the probability of detection evolves when a location is viewed multiple times. The issue of evolving detection probabilities is also overlooked in [10] because of the use of fixed detection probabilities for each seabed type.

In contrast, [9] does address the issue of how the probability of detection changes as a location is viewed multiple times. The assumption made, however, that the probability of failing to detect a target in any single view is independent (and hence those probabilities multiply) is unrealistic. Specifically, this assumption implies that given enough views of a location with a sensor, every target can be detected with probability $1-\epsilon$, for $\epsilon$ arbitrarily small. This belief is false. Many different phenomena (proffered in Sec. II-D) can cause the physics of the problem to simply be absent, and thus prevent the detection of a mine. Sensing a location multiple times in such a situation will not guarantee the detection of a mine's presence.

The previous work with the most accurate assumptions relevant for the underwater mine detection problem are actually found in work focussing on space-based exploration. In [6], the range-dependence of coverage quality - for which detection probability is an apt substitute - is explicitly

\footnotetext{
${ }^{2}$ In this work, the phrase "probability of detection" is assumed to always mean the probability of successfully detecting a target if one were present.
}

accounted for. Moreover, it is assumed that the coverage quality improves to the maximum of any single view of a given location, rather than the multiplicative evolution (of [9]) arising from independence assumptions. We advance the study of the underwater mine detection problem by extending our research in this vein, while also incorporating the effects of seabed type on detection performance.

Additional work [11], [12] has instead examined detection performance without seeking to exploit it for the improvement of AUV path-planning. In [11], detection performance as a function of range was studied for a side-scan sonar system. However, the study was conducted at only a single location (consisting of a single seabed type) with a relatively small number of targets. In [12], a study examining the detection rate of contacts (i.e., clutter and mines together) in SAS imagery of different seabed types was undertaken. Although the results did not establish detection performance of mines, the results did provide anecdotal evidence that supports the hypothesis that seabed type is an important consideration in MCM operations.

Finally, other related work [13] discussed the topic of AUV track-spacing for MCM operations with an eye toward using basic geometry to cover the nadir gap while minimizing the overlap in swaths. This work dealt only with adjusting tracks to alter the geometrical area coverage achieved by a SAS system, but did not address the topic of detection at all.

\section{B. Contribution of Paper}

The main contribution of this work is an algorithm to design the optimal survey route that an AUV should take in order to maximize the mine detection performance in an area. Practical constraints of the application, such as the desire to survey in parallel tracks for imaging purposes, are respected.

This work also provides a metric by which one can assess the detection performance that was or would be achieved for a given mission. Moreover, the work can be used to determine the number of tracks - and equivalently, the amount of time - needed to achieve a certain level of detection performance, rather than using unprincipled estimates. 
In support of these main objectives, several auxiliary contributions are also made.

First, we illuminate several inaccurate assumptions that are commonly made about the problem, and explain the reasons why they are flawed. In particular, we argue that mine detection performance must explicitly account for range and seabed-type dependence.

Second, we address the manner in which the detection probability should evolve when an area of seabed is observed with multiple views.

Third, using an enormous database of real SAS data collected at sea, we develop a model for mine detection probability as a function of range in different seabed types. Although the resulting model is for a specific system (i.e., detection algorithm and SAS sensor) the method is general in that an identical approach can be taken by other researchers with their own systems.

The most substantive practical benefit of this work is that the proposed approach can be immediately applied to systems conducting MCM operations at sea. Because the entire route of the AUV can still be designed before deployment, no additional onboard processing or adaptive capabilities are required of the AUV. That is, there is no obstacle to adopting the proposed track-design approach instead of a standard, uniform ladder survey.

\section{Organization of Paper}

The remainder of this paper is organized in the following manner. Sec. II discusses underwater mine detection and includes a presentation of a detection algorithm and its results on real SAS data. This detection work is then exploited in the proposed AUV track-spacing algorithm in Sec. III. Experimental results of the track-spacing algorithm using real data collected at sea are shown in Sec. IV. Concluding remarks and directions for future work are made in Sec. V.

\section{UNDERWATER MINE DETECTION}

\section{A. Overview}

Sonar can provide high-resolution imaging of underwater environments, which is useful for the detection of mines on the seabed. AUVs equipped with side-looking sonar - SAS or lower-resolution side-scan sonar - is increasingly used for this purpose. Usually, such AUVs are equipped with two sonars, one on the port side and one on the starboard side. These sensors image in a direction orthogonal to the motion of the AUV. Because of the geometry of the problem, a dead zone - from the AUV's nadir up to a certain range on either side - between the two sonar swaths will lack sonar coverage. The standard AUV survey path used in practice is a series of equidistant parallel tracks such that the swaths of consecutive tracks interleave, resulting in coverage for the entire area of interest.

Once the sonar data has been collected and processed into imagery, a mine detection algorithm will be applied. Typically, these detection algorithms search for highlight-shadow patterns characteristic of mines [14], [15]. The highlight is the result of the acoustic echo from the mine itself, while the shadow that is cast is due to the geometry between the mine (and specifically its height above the seafloor) and the grazing angle of the transmitted signal. Some examples of mines in SAS imagery are shown in Fig. 1.

\section{B. Detection Algorithm}

We argue that the probability of detecting a mine should be a function of both range and seabed type. To obtain a realistic model of this mine detection probability, we use real SAS data collected at sea. However, because the detection algorithm is not the main focus of this paper, we describe the method in a cursory manner here.

In this work, a cascaded detection algorithm composed of two stages is employed. In the first stage, a detection algorithm from [15] is applied to the SAS imagery as the initial detector; this algorithm correlates a template, consisting of a generic highlight-shadow pattern characteristic of mines, with the scene image. The resulting correlation at each location in the image is treated as the detection score $\delta_{1}$. Each alarm with a detection score above a given low threshold, $\tau_{1}$, is then passed to the second stage of the cascaded detector.

In the second stage, two templates - a highlight template and a separate shadow template - are correlated with the image chip of each alarm. For each alarm, the maximum of the sum of these correlations is taken to be the detection score $\delta_{2}$. All alarms for which this second detection score is above a given threshold, $\tau_{2}$, is retained; the other alarms are discarded.

It has been found that applying the second stage to the detection process removes many false alarms without greatly eliminating many mines (whereas opting for a single-stage approach in which the first threshold is higher would remove many mines). In practice, the detection thresholds $\tau_{1}$ and $\tau_{2}$ are set to achieve a desired alarm density.

\section{Detection Results}

In April-May 2008, the NATO Undersea Research Centre (NURC) conducted the Colossus II sea trial in the Baltic Sea off the coast of Latvia. During this trial, high-resolution sonar data was collected by the MUSCLE autonomous underwater vehicle (AUV). This AUV is equipped with a $300 \mathrm{kHz}$ sonar with a $60 \mathrm{kHz}$ bandwidth that can achieve an along-track image resolution of approximately $3 \mathrm{~cm}$ and an across-track image resolution of approximately $2.5 \mathrm{~cm}$. The sonar data was subsequently processed into SAS imagery.

The above cascaded detection algorithm was applied to this data set of over 9,000 SAS images spanning a total area of approximately $50 \mathrm{~km}^{2}$. Targets (i.e., mines) were laid before some of the surveys, so ground-truth information is available, from which we can determine the probability of detection of the system (SAS sensor and cascaded detection algorithm). The targets were laid in three different seabed types - flat mud, flat sand, and sand ripples — which allows the detection performance as a function of seabed type (in addition to range) to be assessed. In total, there were approximately 200 target detection opportunities in each of the three seabed types. 


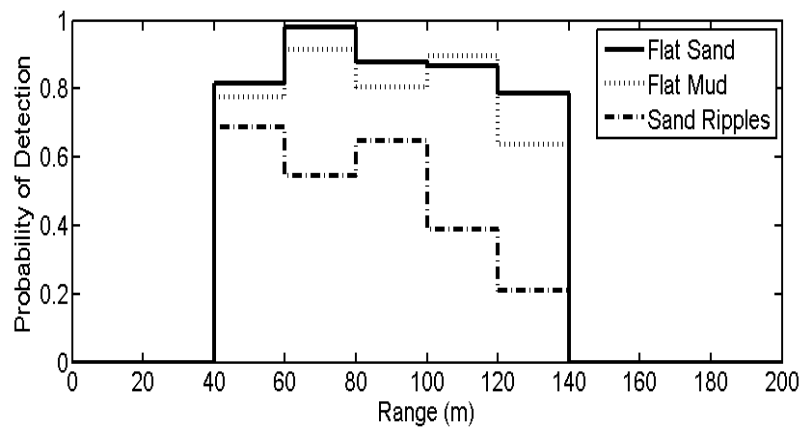

Fig. 2. Probability of detection as a function of range and seabed type.

The results of the detection algorithm in the three seabed types as a function of range are summarized in Fig. 2. (Range bins of $20 \mathrm{~m}$ were used in the calculations.) It should be noted that if lower detection thresholds $\left(\tau_{1}\right.$ and $\left.\tau_{2}\right)$ were used, the false alarm rate would increase but the probability of detection would still not always reach unity.

It is these detection results that will drive the AUV trackspacing experiments in Sec. IV.

\section{Detection Improvement with Multiple Views}

The assumption made in [9] is that the probability of failing to detect a target in any single view is independent, and hence those probabilities multiply. Specifically, this assumption implies that given enough views of a location with a sensor, every target can be detected with probability $1-\epsilon$, for $\epsilon$ arbitrarily small.

However, many different phenomena can cause the physics of the problem to simply be absent, and thus prevent the detection of a mine. Sensing a location in such an instance multiple times will not guarantee the detection of a mine's presence.

For example, a mine can be fully or partially buried such that it casts no shadow. Additionally, a mine can be located in a scour pit, which would alter the characteristic highlightshadow signature of the mine. Alternatively, a mine can be composed of a material for which the frequency of the imaging sonar is incompatible for detection purposes. And finally, a mine can be obscured by the shadows of large rocks or other bathymetric anomalies; example SAS imagery of this scenario is shown in Fig. 3.

For these reasons, we adopt the convention employed in [6] for when a location is interrogated multiple times. Specifically, it is assumed that the probability of detecting a mine if one is present improves to the maximum of any single view of a given location.

\section{AUV TRACK-SPACING}

The adherence to traversing parallel tracks in MCM operations is partly because the collected raw data is subsequently processed into imagery (e.g., SAS imagery), for which such data is preferable. Thus, the crux of the path-planning task becomes how to design the spacing of (parallel) tracks. We address this objective, noting that the removal of the

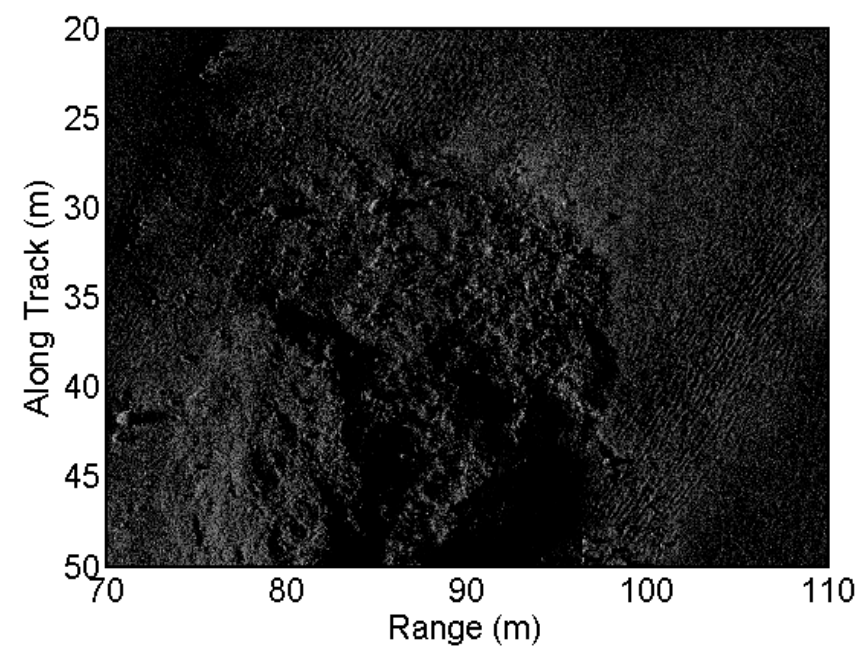

Fig. 3. SAS image with a bathymetric anomaly, the shadows of which would prevent - even with multiple views - the detection of mines.

constraint permitting only parallel tracks does not induce substantive complications.

\section{A. Objective Function}

The objective function we seek to optimize for the pathplanning work is defined to be the reward accrued from performing a track; this reward is simply the difference of the benefit and the cost of the track. The benefit of the track is defined to be the increase in the mean probability of detection over the entire mission area of interest.

The cost of the track is defined to be the energy costs (e.g., battery) - in units adapted to be consistent with those of the benefit - required to perform the track, which are proportional to the distance of the track (plus transit distance between tracks). This formulation naturally leads to an intuitive termination criterion: perform tracks as long as they would produce a positive reward (i.e., the benefit exceeds the cost).

In practice, the relationship between the benefit and cost of a track depends on the specific mission objectives and must be specified by the controller of the AUV. Defining this relationship will essentially determine when the algorithm should terminate, and hence the number of tracks that can be undertaken.

Alternatively, it can be assumed that the AUV has sufficient energy to achieve a desired mean detection level; in this case, the cost can be ignored so that the reward is equivalent to the benefit. This situation is the realistic one encountered at sea when an area must be surveyed to a certain detection level, regardless of the length of time needed. To maintain the focus on the general performance of the track-spacing algorithm itself, as opposed to effects of problem-specific costs, we assume we are operating in this regime hereafter.

Thus, the focus of the problem lies solely on determining the best track-centerlines for the AUV to traverse. 


\section{B. Track-Spacing Algorithm}

The proposed track-spacing algorithm consists of a myopic (greedy) search for the best tracks. At each iteration, the expected benefit (and cost) of every possible track is calculated. The track that maximizes the expected reward (or benefit when costs are ignored) at each iteration is chosen and added to the set of tracks to traverse. The benefit is calculated based on the probability of detection as a function of range and seabed type ( $c f$. Fig. 2) for the area covered by the track's swaths.

When it is determined that the objective will have been met with the set of selected tracks - i.e., that the mean probability of detection over the mission area exceeds the desired level - the algorithm terminates. Importantly, this entire process can be undertaken before the AUV is even deployed.

Because of the greedy approach employed, the set of selected tracks is necessarily ordered in terms of decreasing benefit. Even so, it is not guaranteed that a (local) optimum of the objective function has been reached. To further improve upon the initial set of tracks selected, and to compensate for the adverse effects of the greedy approach, a small geographical displacement of each track is considered. One at a time, we consider the result if a given track is shifted to the port or starboard side one spatial increment. If the objective function increases, the displaced track replaces the previous version of the track. This iterative process continues sequentially for all tracks, one at a time, until no such new shift improves the objective function. This adjustment stage can be thought of as improving closer to the (local) optimum.

A second optional adjustment can also be made. The set of selected tracks are ordered in terms of decreasing benefit. However, because we possess this entire set of tracks before any track is traversed, the set of tracks can easily be resorted in terms of geography so that the total transit distance (i.e., between the end and beginning of consecutive tracks) is minimized. Although this rearrangement will not impact the benefit of the set of the tracks, it will minimize energy consumption costs.

It should be noted that the exhaustive, brute-force track search that comprises the main portion of the algorithm is not as computationally demanding as one may expect. This fact is because the swath coverage of many tracks will not intersect with the swath coverage of most other tracks. As a result, the benefit of certain tracks can be calculated once and saved. Only the tracks for which the coverage of the previously selected track intersects must be recomputed in a given iteration. The computational savings from exploiting this insight increase proportional to the size of the mission area.

In summary, the proposed track-spacing algorithm consists of three stages. First, an exhaustive greedy search is conducted until the detection criterion is reached. Second, minor track adjustments via small centerline displacements are considered. Third, the set of tracks is reordered in terms of geography.

\section{Benefit Calculation}

The benefit of a track is defined in terms of the mean increase in the probability of detection over the mission area. This benefit can be obtained by calculating the increase in the probability of detection at each location in a track. Specifically, the benefit at a location $(x, y)$ covered by a track's swath is $B(x, y)=p(d \mid(x, y))-p^{\prime}(d \mid(x, y))$, where $p^{\prime}(d \mid(x, y))$ and $p(d \mid(x, y))$ are the probabilities of detection at the given location before and after the new track is run, respectively.

Let $s(x, y)$ be the seabed type at location $(x, y)$, and $r(x, y)$ be the range from the AUV (i.e., track centerline) to the same location. For the location $(x, y)$ covered by a track, the probability of detection will be updated according to

$$
p(d \mid(x, y))=\max \left\{p^{\prime}(d \mid(x, y)), p(d \mid s(x, y), r(x, y))\right\},
$$

where $p(d \mid s(x, y), r(x, y))$ is the probability of detection at range $r(x, y)$ from the model corresponding to seabed type $s(x, y)$ in Fig. 2. The 'maximum' operator arises in (1) because we assume that the probability of detection at a point that is viewed multiple times is the maximum from among those individual views.

The above assumes that the true seabed-type at every location, $s(x, y)$, is known a priori over the entire mission area. When this is the case, the true benefit of every track can be calculated readily. This a priori seabed-type information can be obtained, for instance, from previous surveys conducted in the area.

When the true seabed-type is not known, $p(d \mid s(x, y), r(x, y))$ in (1) will be replaced by an approximation, $\widetilde{p}(d \mid \boldsymbol{\pi}(x, y), r(x, y))$, that is based on the belief that one holds about the seabed, which is contained in the ambiguous quantity $\boldsymbol{\pi}(x, y)$.

This approximation can be formulated in terms of a set of prior probabilities on each potential seabed type. Thus, when calculating the expected benefit of a track when the true seabed is not known, rather than using the appropriate probability of detection model given in Fig. 2, one would instead weight each model by the prior probability of each seabed type,

$$
\widetilde{p}(d \mid \boldsymbol{\pi}(x, y), r(x, y))=\sum_{s_{i} \in \mathcal{S}} \pi_{s_{i}}(x, y) p\left(d \mid s_{i}, r(x, y)\right),
$$

where $p\left(d \mid s_{i}, r(x, y)\right)$ is the probability of detection at range $r(x, y)$ if the seabed type is $s_{i}$ from the set of possible seabed types $\mathcal{S}$, and $\pi_{s_{i}}(x, y)$ is the prior probability of encountering seabed type $s_{i}$ at location $(x, y)$. The prior probabilities are non-negative and sum to unity. In this work, $\mathcal{S}=\{$ flat sand, flat mud, sand ripples $\}$.

In the event that no knowledge about the seabed is possessed, one can specify an uninformative prior in which equal prior probabilities are assumed for each possible seabed type everywhere.

In practice, one will usually possess at least some knowledge of the seabed types to be encountered. Additionally, 
it should be noted that for post-mission analysis objectives, one will almost certainly know the composition of the seabed since the data will have been collected.

\section{EXPERIMENTAL RESULTS}

\section{A. Data Set}

To evaluate the proposed adaptive track-spacing algorithm, we conduct experiments using real data collected during the sea trial described in Sec. II-C. Specifically, the mission area on which the experiments are applied is taken to be a nearly $4 \mathrm{~km}^{2}$ area of seabed surveyed during the trial. We exploit the manual ground-truthing result of this area (from [16]) in our experiments here. The mission area is composed of flat sand and sand ripples, as shown by the (ground-truth) seabed map in Fig. 4.

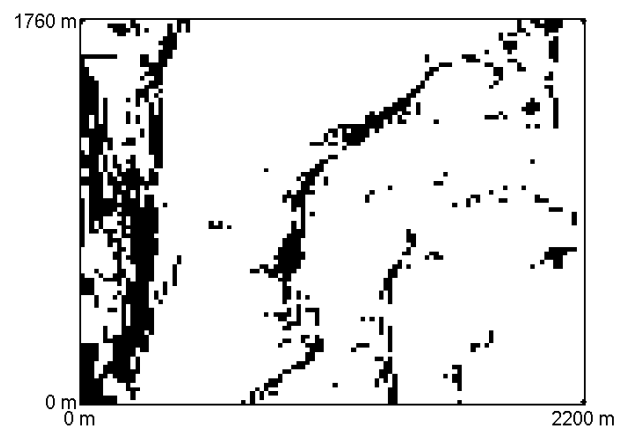

Fig. 4. Seabed map for the mission area in the Baltic Sea used in the experiments, where white areas correspond to flat sand and black areas correspond to sand ripples.

\section{B. Experimental Set-up}

The objective of the proposed track-spacing algorithm is to determine the set of tracks that will achieve the highest mean probability of detection over the entire mission area.

(It should be noted that the only image data that exists is that associated with the survey tracks actually run at sea. However, to evaluate the proposed method via the experiments here, re-creating the imagery for a different potential track is not necessary because, at this stage, obtaining the probability of detection values requires only the seabed type (and range), not the actual image data.)

In these experiments, we compare three different trackspacing methods on the area of seabed described in Sec. IVA. Specifically, we consider two versions of the proposed adaptive-spacing algorithm and one baseline method that employs uniformly-spaced tracks.

The adaptive-spacing cases differ in the knowledge that is assumed about the seabed. In one case, it is assumed that the composition of the seabed is known a priori. In the second case, it is assumed that no knowledge of the composition of the seabed is possessed.

For this latter case, an uninformative prior (as described in Sec. III-C) is assigned to the probability of encountering each of three seabed types - flat sand, flat mud, and sand ripples - everywhere in the mission area. That is, in calculating the expected benefit of a track via $(2), \pi_{s_{i}}(x, y)=1 / 3$ for each $s_{i} \in \mathcal{S}$.

For each of the three approaches considered, a set of $N=$ 18 (vertical) tracks was selected. This value of $N$ was used because it was the number of tracks needed to attain the desired mean probability of detection for the case of adaptive spacing with no seabed knowledge.

The above experiments are meant to demonstrate the utility of the proposed adaptive-spacing algorithm for optimizing detection performance in future missions for which an AUV will be deployed. However, the proposed overall framework - how the probability of detection evolves with multiple views and as a function of range and seabed type - can also be used to assess the level of detection achieved by already completed missions. To this end, the detection performance resulting from the tracks ( 8 horizontal and 10 vertical) that were actually run during the Colossus II sea trial in the mission area under study is also presented.

\section{Results}

The main experimental comparison is among the three approaches used to determine the best set of AUV tracks. Maps that qualitatively show the final probability of detection everywhere in the mission area resulting from the selected set of tracks for each of the three approaches are shown in Fig. 5; this figure also shows the level of detection achieved by the tracks that were actually run during the Colossus II sea trial.

In addition to the maps of Fig. 5, the performance of the three approaches is also summarized quantitatively, in terms of the mean probability of detection over the entire mission area, in Table I.

To demonstrate the value of the stage of the proposed approach that involves track-centerline shifts, we present this mean probability of detection over the mission area, with and without the final track-centerline shifts. (For the cases in which the seabed was assumed known and unknown, five and three of the 18 tracks were chosen to be displaced one spatial increment, respectively.)

\section{TABLE I}

MEAN PROBABILITY OF DETECTION OVER THE MISSION AREA

\begin{tabular}{|c|c||c|c|}
\hline $\begin{array}{c}\text { SPACING } \\
\text { METHOD }\end{array}$ & \multicolumn{1}{|c||}{ SEABED } & WITHOUT & WITH \\
CENTERLINE SHIFTS
\end{tabular}

As can be seen from the table, the proposed adaptive track-spacing approach achieves a higher mean probability of detection than the baseline approach that employs uniform track-spacing. Importantly, the adaptive approach performs better than the uniform spacing approach even when it is assumed that no knowledge of the seabed is possessed.

Table I provides only a coarse summary measure of performance. To more finely assess the implications of the different approaches, the results are presented in an alternative format 

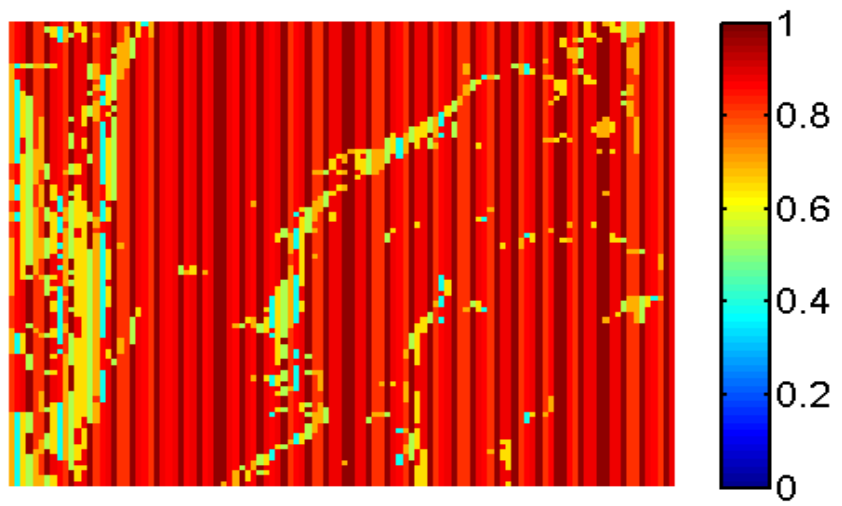

(a)
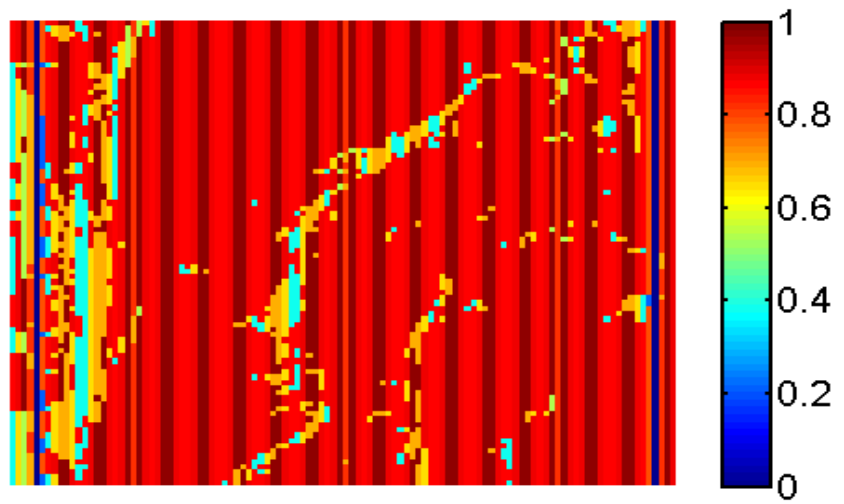

(c)

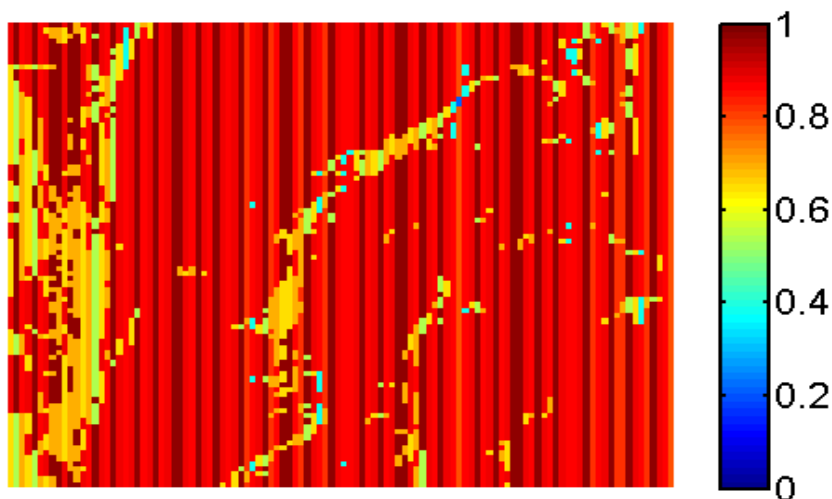

(b)

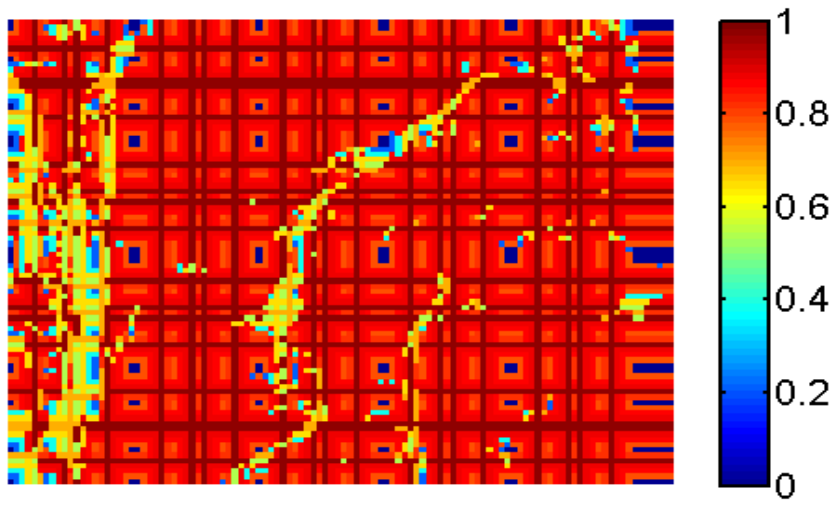

(d)

Fig. 5. Final probability of detection maps from performing a certain set of 18 tracks: using adaptively-spaced tracks (proposed method) when the seabed is (a) unknown, and (b) known; (c) using uniformly-spaced tracks; (d) using the tracks actually run during the Colossus II trial.

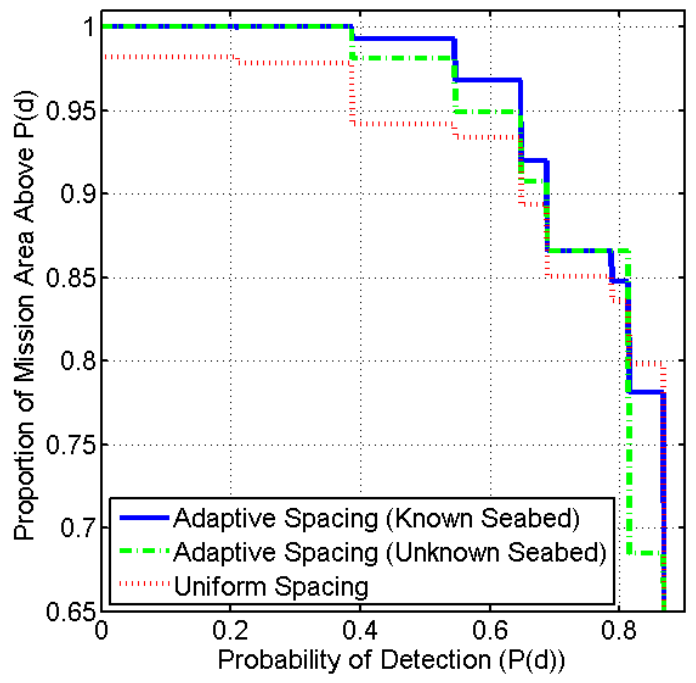

Fig. 6. Proportion of the mission area that is above any probability of detection for the three cases considered.

in Fig. 6. Specifically, this figure shows the proportion of the mission area that has a final probability of detection that is above a given value.

This method of assessment is valuable because in practice it is important to know that everywhere in the mission area has been covered to a certain probability of detection. For example, at the natural threshold of a probability of detection of 0.5 , it can be seen from the figure that $5.80 \%$ of the area has not been covered to this level when employing uniform track-spacing. In comparison, only $0.69 \%$ and $1.90 \%$ of the area has not been covered to this level when employing the proposed adaptive spacing, with or without seabed knowledge, respectively.

Next, to demonstrate the value of the final stage of the proposed approach that involves reordering tracks geographically, we present the transit distance for each of the approaches with and without this sorting step, in Table II.

TABLE II

TOTAL TRANSIT DISTANCES (IN KILOMETERS)

\begin{tabular}{|c|c|c|c|}
\hline SPACING & & WITHOUT & $\overline{\text { WITH }}$ \\
\hline METHOD & SEABED & \multicolumn{2}{|c|}{ TRACK SORTING } \\
\hline UNIFORM & - & \multicolumn{2}{|c|}{1.98} \\
\hline ADAPTIVE & UNKNOWN & 8.14 & 2.04 \\
\hline ADAPTIVE & KNOWN & 15.82 & 1.96 \\
\hline
\end{tabular}

The transit distance is defined here to be the sum of the distances from the end of each track to the beginning of the 


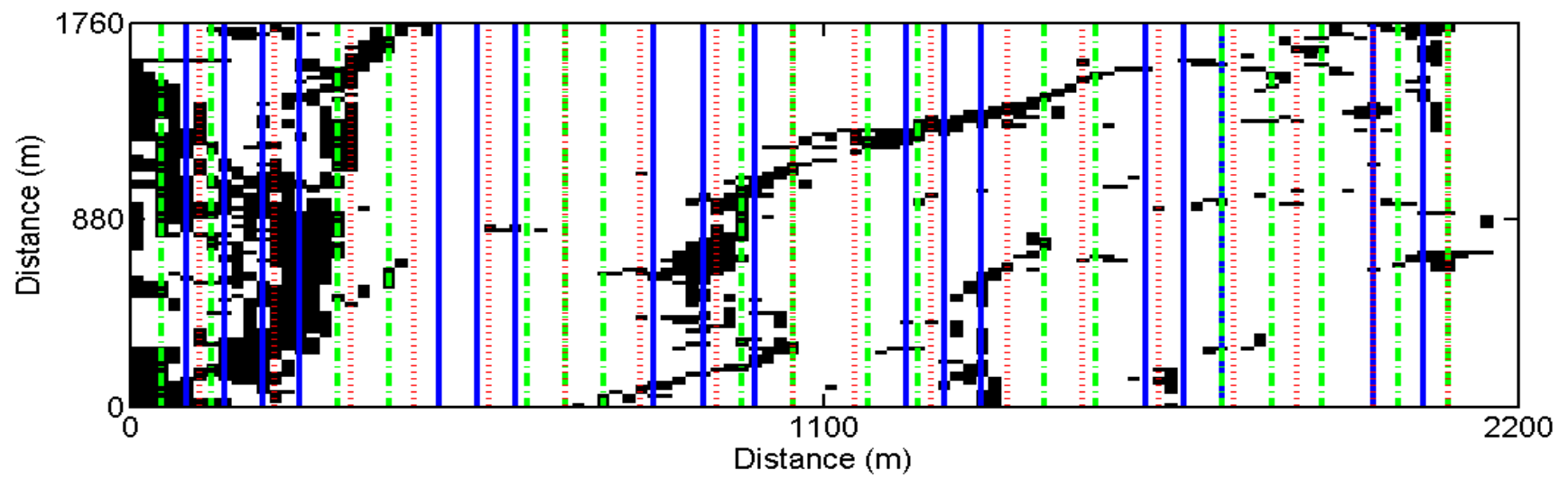

Fig. 7. Selected track centerlines for each of the three track-spacing methods considered. The correspondence of methods to line colors and types are the same as that in Fig. 6. (Note that the figure is stretched horizontally to more clearly display the tracks.)

subsequent track.

As can be seen from the table, including the geographical sorting of tracks in the algorithm greatly reduces the transit distance, which in turn would allow resources (e.g., battery life) to be conserved.

Finally, we show the locations of the actual tracks selected by the three different approaches - from which all of the preceding results were obtained - overlaid on the mission area's seabed map, in Fig. 7.

\section{CONCLUSION}

This work addressed the task of designing the optimal survey route that an AUV should take in MCM operations. In the process, several commonly made - but inaccurate assumptions about the problem were also raised and refuted.

An elegantly simple algorithm to design the optimal survey route that an AUV should take was proposed. The work provides a metric - the mean probability of detection - by which one can quantify the detection performance achieved in a past mission. Alternatively, the work can be used to determine the number and location of tracks needed to achieve a certain level of detection performance in a future mission.

The proposed approach can be immediately applied to systems conducting MCM operations at sea. Because the entire route of the AUV can still be designed before deployment, no additional onboard processing or adaptive capabilities are required of the AUV. That is, there is no risk in adopting the proposed track-design approach instead of a standard, uniform ladder survey. Therefore, in an upcoming NURC sea trial, the proposed adaptive track-spacing algorithm will be employed for determining the AUV's data-collection route.

Other future work will seek to refine the probability of detection models of Fig. 2, using more data, because intuition suggests that the curves therein should be smoother. Additional work will be devoted to developing similar models for other seabed types, such as posidonia fields. The dependence of the probability of detection models on other features such as bathymetric anomalies and the size and orientation of sand ripples - will also be investigated.

\section{ACKNOWLEDGMENTS}

The author would like to thank Johannes Groen of NURC for providing the results of the first stage of the detection algorithm.

\section{REFERENCES}

[1] C. Warren, "A technique for autonomous underwater vehicle route planning," IEEE J. Oceanic Engineering, vol. 15, pp. 199-204, 1990.

[2] C. Pêtrès, Y. Pailhas, P. Patrón, Y. Petillot, J. Evans, and D. Lane, "Path planning for autonomous underwater vehicles," IEEE Trans. Robotics, vol. 23, no. 2, pp. 331-341, 2007.

[3] D. Kruger, R. Stolkin, A. Blum, and J. Briganti, "Optimal AUV path planning for extended missions in complex, fast-flowing estuarine environments," in IEEE International Conference on Robotics and Automation (ICRA), 2007, pp. 4265-4270.

[4] M. Soulignac, P. Taillibert, and M. Rueher, "Time-minimal path planning in dynamic current fields," in IEEE International Conference on Robotics and Automation (ICRA), 2009, pp. 2473-2479.

[5] R. Knight and B. Smith, "Optimally solving nadir observation scheduling problems," in Proc. 8th International Symposium on Artificial Intelligence, Robotics and Automation in Space (iSAIRAS), 2005.

[6] D. Gaines, T. Estlin, and C. Chouinard, "Spatial coverage planning for a planetary rover," in IEEE International Conference on Robotics and Automation (ICRA), 2008, pp. 614-620.

[7] H. Choset and P. Pignon, "Coverage path planning: The boustrophedon cellular decomposition," in International Conference on Field and Service Robotics, 1997.

[8] J. Stack and C. Smith, "Combining random and data-driven coverage planning for underwater mine detection," in Proc. IEEE OCEANS, 2003, pp. 2463-2468.

[9] A. Rajala and D. Edwards, "Allocating AUVs for mine map development in MCM," in Proc. IEEE OCEANS - Asia Pacific, 2007.

[10] N. L. Bouffant, P. Pidsley, J. Malkasse, and F. Florin, "Automatic MCM mission control for AUV systems," in Proc. IEEE OCEANS, 2005, pp. 930-936.

[11] K. Bryan, "Analysis of the AUVPET07 trial," NATO Undersea Research Centre, La Spezia, Italy, Tech. Rep. NURC-FR-2009-005, 2009.

[12] J. Groen, E. Coiras, and D. Williams, "Detection rate statistics in synthetic aperture sonar images," in Proc. Intl. Conf. \& Exh. Underwater Acoustic Measurements (UAM), 2009, pp. 367-374.

[13] P. Hagen and R. Hansen, "Area coverage rate of synthetic aperture sonars," in Proc. IEEE OCEANS, 2007.

[14] G. Dobeck, J. Hyland, and L. Smedley, "Automated detection/classification of seamines in sonar imagery," in Proc. SPIE International Society of Optics, vol. 3079, 1997, pp. 90-110.

[15] J. Fawcett, A. Crawford, D. Hopkin, V. Myers, and B. Zerr, "Computer-aided detection of targets from the CITADEL trial Klein sonar data," Defence R \& D Canada - Atlantic, Canada, Tech. Rep. DRDC Atlantic TM 2006-115, 2006.

[16] D. Williams, "Bayesian data fusion of multiview synthetic aperture sonar imagery for seabed classification," IEEE Trans. Image Processing, vol. 18, no. 6, pp. 1239-1254, 2009. 